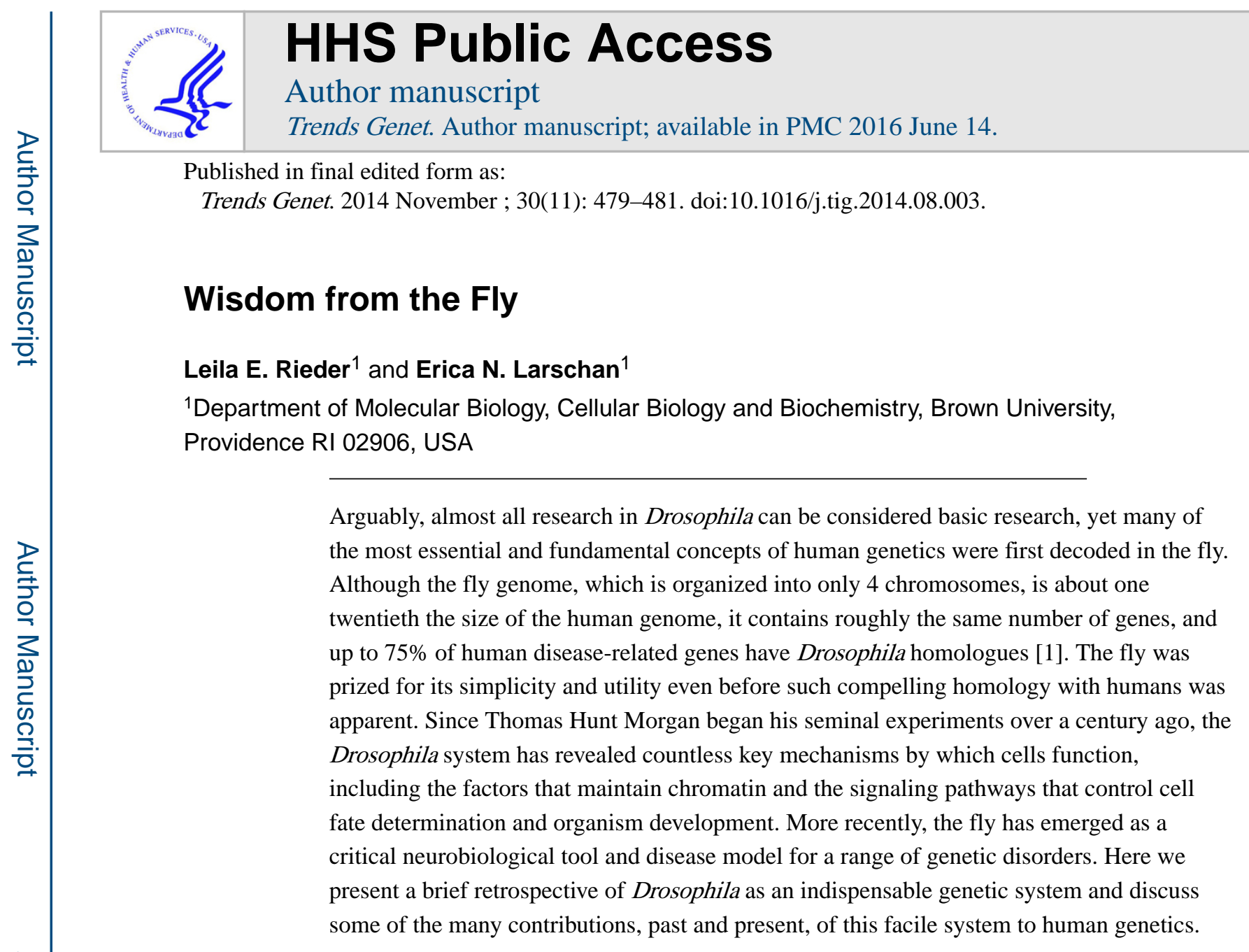

\title{
Chromatin maintenance and regulation
}

The control and maintenance of chromatin state is a critical to the function of all cells, and much of our knowledge of how genes are regulated by chromatin came from the discovery of position-effect variegation (PEV) by H. J. Muller in 1930. His observation that the expression of a phenotype could be affected by the placement of a gene within different chromatin contexts led to the identification of many enhancers (E(var)) and suppressors $(\mathrm{Su}(\mathrm{var}))$ of variegation via genetic screens in the fly. These proteins are often conserved, such as heterochromatin protein-1 (HP-1), and are responsible for the establishment and maintenance of chromatin states. Furthermore, the discovery of $\mathrm{Su}(\mathrm{var})$ and $\mathrm{E}(\mathrm{var})$ mutations led to the first models of a balanced chromatin state that relies on both activators and repressors for proper function [2].

Critical clues into how chromatin state is initiated and maintained arrived in the 1960s with the identification of polycomb group proteins $(\mathrm{PcG})$ and trithorax group proteins $(\operatorname{Trx} \mathrm{G})$ in Drosophila. Discovery began through a series of Drosophila mutagenesis screens that identified key regulators of the Hox genes, which encode a series of homeotic transcription

Corresponding Author: Larschan, E (Erica_Larschan@Brown.edu).

Publisher's Disclaimer: This is a PDF file of an unedited manuscript that has been accepted for publication. As a service to our customers we are providing this early version of the manuscript. The manuscript will undergo copyediting, typesetting, and review of the resulting proof before it is published in its final citable form. Please note that during the production process errors may be discovered which could affect the content, and all legal disclaimers that apply to the journal pertain. 
factors required for fly embryo patterning and development. The conserved PcG and TrXG complexes, repressors and activators of chromatin, respectively, are responsible for the precise genetic regulation of the Hox genes through deposition of a series of chromatin marks.

While early research into PcGs and TrxGs suggested their role in homeotic gene control in both Drosophila and mammalian systems, recent genome-wide studies have revealed that these complexes regulate myriad critical genes throughout the genome in metazoa and some plants [3]. PcGs and TrxGs have complex roles in development, cell differentiation, and cell reprogramming because they establish epigenetic "memory" of heritable chromatin states. It is therefore not surprising that the misregulation of these proteins is implicated in a multitude of human disease conditions, most notably cancers [4, 5]. The Drosophila system is also a well-suited model for study of the connection between PcGs, TrxGs and tumorigenesis. For example, Drosophila is being used to determine how PcGs target specific oncogenes for regulation during cell fate determination [6].

\section{Signaling pathways in development and disease}

Just as Muller observed PEV in Drosophila as a curious variegated eye phenotype, John Dexter first characterized mutant "notched" wing phenotypes a hundred years ago, spurring a century of investigation into the underlying signaling pathways. The Notch pathway is highly conserved within metazoa and involves a series of receptors, ligands, and transcription factors important in cell-cell communication and essential for cell fate determination and embryonic development.

Another essential group of signaling pathways was first identified through phenotypic observations of the striking loss of wings or halteres caused by a hypomorphic recessive mutation in the wingless gene. The conserved wingless (Wnt) signal transduction pathways are involved in a variety of processes, including vertebrate brain, kidney, limb, and gut development. Components of the highly conserved Bone Morphogenetic Protein (BMP) signaling pathway were also initially identified in Drosophila. Early studies into the BMP pathway focused on embryo patterning and development, including the early establishment of patterning along the dorsal-ventral body axis. The BMP pathway, along with the related Activin/TGF- $\beta$ pathway, is now known not only to induce the formation of bone, but also to affect a multitude of cellular functions in homeostasis and during development.

Misregulation of any of the Notch, Wnt, or BMP/TGF- $\beta$ pathways is implicated in a wide variety of human genetic diseases and cancers [7, 8]. For example, a single point mutation in a BMP receptor is associated with fibrodysplasia ossificans progressiva, a rare autosomal dominant disorder in which muscle and connective tissue are slowly replaced by bone [8]. This disease is currently being studied in a Drosophila model [9]. As we extend our understanding of how the signals transduced by each pathway are regulated, the list of associated human genetic diseases will continue to grow. 


\section{Drosophila as an essential neurobiological model}

The fly has historically facilitated the identification of critical cellular mechanisms and laid the foundation of human genetics. Yet the utility of Drosophila is not limited to developmental cellular biology: the fly has recently emerged as an incredibly useful neurobiological model. Drosophila is an attractive system in which to study neurobiology for several key reasons. First, there are significant similarities between the fly and human brains, including the expression of important regulatory genes [10]. Perturbed fly behaviors, the output of altered brain function, are well characterized and easily identified, quantified, and genetically traced. Second, the Drosophila genome is less complex than those of mammalian models, partially due to the presence of smaller gene families. The genetic simplicity of the fly allows for the identification of interactions that might be masked by redundancy in more complex vertebrate systems. Finally, the unparalleled extensive genetic toolbox already available in Drosophila facilitates and speeds discovery.

Because of the extensive genetic tractability and relevance of the fly, it has already been employed to model countless neurodegenerative diseases, including Parkinson's, Alzheimer's, and Huntington's diseases [11], and amyotrophic lateral sclerosis (ALS) [12]. Further, the creation of fly models for human neurobiological diseases allows for large-scale pharmacological testing and drug discovery [11]. These applications are particularly useful, as they can be prohibitively expensive and lengthy in vertebrate models.

\section{Genetic tools in Drosophila}

Historically, relatively simple phenotypic observations in Drosophila have lead to the discovery of complex and conserved genetic mechanisms. The fly will doubtlessly continue to be an indispensable tool for genetic research, especially given that most recently discovered genetic tools are easily modified to work in the fly; RNA interference for transcript knock down, and TALEN and CRISPR tools for gene knock out were all quickly adapted for use in Drosophila. These newer tools, coupled with the power of the classical high throughput genetic screen, a relatively simple approach in Drosophila, serve to keep the fly highly relevant to the study of human genetics. Additionally, the simplicity of the fly genome compared to complex vertebrate genomes and the large amount of available public data [13] allow for strong computational biology and genome-wide association studies.

In summary, Drosophila has historically been an indispensible genetic tool. In addition to numerous other contributions, the fly has revealed mechanistic insights into the conserved processes of chromatin maintenance and signal transduction. It is also apparent that the Drosophila system is already a powerful neurobiological tool, and the list of available resources continues to expand at a rapid pace [14]. It is highly likely that future discoveries in the fly will prove to be just as essential to our understanding of human genetics as those early observations of curious phenotypes. 


\section{References}

1. Reiter LT, Potocki L, Chien S, Gribskov M, Bier E. A systematic analysis of human diseaseassociated gene sequences in Drosophila melanogaster. Genome research. 2001; 11:1114-1125. [PubMed: 11381037]

2. Pillus L. An acquired state: epigenetic mechanisms in transcription. Current opinion in cell biology. 1992; 4:453-458. [PubMed: 1497916]

3. Kassis JA, Brown JL. Polycomb group response elements in Drosophila and vertebrates. Advances in genetics. 2013; 81:83-118. [PubMed: 23419717]

4. Zhang C, Liu B, Li G, Zhou L. Extra sex combs, chromatin, and cancer: exploring epigenetic regulation and tumorigenesis in Drosophila. Journal of genetics and genomics = Yi chuan xue bao. 2011; 38:453-460. [PubMed: 22035866]

5. Valk-Lingbeek ME, Bruggeman SW, van Lohuizen M. Stem cells and cancer; the polycomb connection. Cell. 2004; 118:409-418. [PubMed: 15315754]

6. Classen AK, Bunker BD, Harvey KF, Vaccari T, Bilder D. A tumor suppressor activity of Drosophila Polycomb genes mediated by JAK-STAT signaling. Nature genetics. 2009; 41:1150 1155. [PubMed: 19749759]

7. Clevers H, Nusse R. Wnt/beta-catenin signaling and disease. Cell. 2012; 149:1192-1205. [PubMed: 22682243]

8. Bandyopadhyay A, Yadav PS, Prashar P. BMP signaling in development and diseases: a pharmacological perspective. Biochemical pharmacology. 2013; 85:857-864. [PubMed: 23333766]

9. Le VQ, Wharton KA. Hyperactive BMP signaling induced by ALK2(R206H) requires type II receptor function in a Drosophila model for classic fibrodysplasia ossificans progressiva. Developmental dynamics: an official publication of the American Association of Anatomists. 2012; 241:200-214. [PubMed: 22174087]

10. Thor S. The genetics of brain development: conserved programs in flies and mice. Neuron. 1995; 15:975-977. [PubMed: 7576663]

11. Nichols CD. Drosophila melanogaster neurobiology, neuropharmacology, and how the fly can inform central nervous system drug discovery. Pharmacology \& therapeutics. 2006; 112:677-700. [PubMed: 16935347]

12. Gama Sosa MA, De Gasperi R, Elder GA. Modeling human neurodegenerative diseases in transgenic systems. Human genetics. 2012; 131:535-563. [PubMed: 22167414]

13. Celniker SE, Dillon LA, Gerstein MB, Gunsalus KC, Henikoff S, Karpen GH, Mod EC. Unlocking the secrets of the genome. Nature. 2009; 459:927-930. [PubMed: 19536255]

14. Jenett A, Rubin GM, Ngo TT, Shepherd D, Murphy C, Dionne H, Zugates CT. A GAL4-driver line resource for Drosophila neurobiology. Cell reports. 2012; 2:991-1001. [PubMed: 23063364] 


\section{Table 1}

Classic and pertinent Drosophila genetics publications.

\section{John S. Dexter}

"The analysis of a case of continuous variation in Drosophila by a study of its linkage relations," American Naturalist.

Included a description of the mutant "notched" phenotype. Morgan later identified the alleles in 1917.

1915 Thomas Hunt Morgan

Mechanisms of Mendelian Heredity

Demonstrated the principles of "coupling" and "repulsion," later referred to as "linkage

1925 Thomas Hunt Morgan

Nobel prize in physiology or medicine awarded in 1933 for discovering the role of chromosomes in heredity.

The Theory of the Gene

Confirmed Mendel's theory of heredity and established the chromosome as the unit of heredity.

1930 Herman J. Muller

Nobel prize in physiology or medicine awarded in 1946 for production of mutations by X-rays.

"Types of visible variations induced by X-rays in Drosophila," Journal of Genetics.

"The frequency of translocations produced by X-rays in Drosophila," Genetics.

Described early observations of position effect variegation (PEV).

1978 Edward B. Lewis

Nobel prize in physiology or medicine awarded in 1995 for discoveries concerning genetic control of early embryonic development.

"A gene complex controlling segmentation in Drosophila," Nature.

A review, summarizing decades of research, in which Lewis presents his view of the bithorax complex and describes Polycomb as a possible repressor. 